FZJ-IKP(TH)-2003-05

\title{
Investigating scalar meson mixing in nucleon-nucleon and deuteron-deuteron collisions ${ }^{1}$
}

\author{
C. Hanhart \\ IKP, Forschungszentrum Jülich, 52428 Jülich, Germany
}

\begin{abstract}
The advantages to study charge-symmetry breaking and especially the phenomenon of $f_{0}-a_{0}$ mixing in nucleon-nucleon and deuteron-deuteron induced reactions are discussed.
\end{abstract}

\section{INTRODUCTION}

As is clearly demonstrated by the existence of these proceedings, the scalar mesons still are an interesting puzzle with respect to our understanding of QCD. It is striking that, although experimentally well established since many years and seen in various reactions, the fundamental structure of the $f_{0}(980)$ and the $a_{0}(980)$ is still obscure.

Already long ago Achasov argued [1]

- that the almost degenerate isoscalar meson $f_{0}(980)$ and the isovector meson $a_{0}(980)$ should mix and

- that, in the vicinity of the $K \bar{K}$ threshold, this mixing should be enhanced by an order of magnitude compared to usual charge-symmetry breaking (CSB) effects.

However, up to now no unambiguous experimental signal of this predicted behaviour has been seen. We will argue that the ideal reactions to extract information of this CSB matrix element is the production of $f_{0}$ and $a_{0}$ in $N N$ and $d d$ collisions, since these reactions allow to manipulate the isospin of the initial and final states such that CSB leads to unique signals that can be extracted model independently. For details about the planned experimental program we refer to Ref. [2].

The paper is organized as follows: in the next section we review what is known about $a_{0}-f_{0}$ mixing. In section three the particular features of the reactions $N N \rightarrow d X$ and $d d \rightarrow \alpha X$ are discussed in detail. Section four contains a presentation of recent experimental results for the reaction $p p \rightarrow d \bar{K}^{0} K^{+}$that show, that the scalar resonances can indeed be studied in $N N$ collisions close to the kaon threshold. The paper closes with a brief summary as well as an outlook.

\footnotetext{
${ }^{1}$ Invited talk at the international High Energy Physics Workshop Scalar Mesons: an Interesting Puzzle for QCD, May 16-18, 2003, SUNY Institute of Technology, Utica, New York.
} 


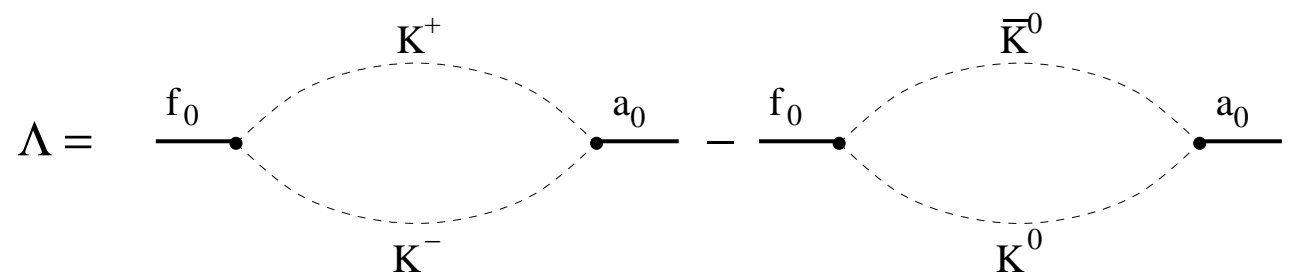

FIGURE 1. Graphical illustration of the leading contribution to the $f_{0}-a_{0}$ mixing matrix element $\Lambda$ defined in Eq. (1).

\section{REMARKS ABOUT $F_{0}-A_{0}$ MIXING}

In Ref. [1] it was demonstrated, that the leading piece of the $f_{0}-a_{0}$ mixing amplitude can be written as ${ }^{2}$

$$
\Lambda=\left\langle f_{0}|T| a_{0}\right\rangle=i g_{f_{0} K \bar{K}} g_{a_{0} K \bar{K}} \sqrt{s}\left(p_{K^{0}}-p_{K^{+}}\right)+\mathscr{O}\left(\frac{p_{K^{0}}^{2}-p_{K^{+}}^{2}}{s}\right),
$$

where $p_{K}$ denotes the modulus of the relative momentum of the kaon pair and the effective coupling constants are defined through $\Gamma_{x K \bar{K}}=g_{x K \bar{K}}^{2} p_{K}$. Obviously, this leading contribution is just that of the unitarity cut of the diagrams shown in Fig. 1 and is therefore model independent. In addition, the contribution shown in Eq. (1) is unusually enhanced between the $K^{+} K^{-}$and the $\bar{K}^{0} K^{0}$ thresholds, a regime of only $8 \mathrm{MeV}$ width. Here it scales as

$$
\sqrt{\frac{m_{K^{+}}^{2}-m_{K^{0}}^{2}}{m_{K^{+}}^{2}+m_{K^{0}}^{2}}} \sim \sqrt{\frac{m_{u}-m_{d}}{m_{u}+m_{d}}}
$$

where $m_{u}$ and $m_{d}$ denote the current quark mass of the up and down quark respectively. This is in contrast to common CSB effects ${ }^{3}$ which scale as $\left(m_{u}-m_{d}\right) /\left(m_{u}+m_{d}\right)$, since they have to be analytic in the quark masses. It is easy to see that away from the kaon thresholds $\Lambda$ returns to a value of natural size. This $\sqrt{s}$ dependence of $\Lambda$ is depicted in Fig. 2. Note, in Eq. (11) electro magnetic effects were neglected for they are also subleading [1].

So far, little is known about the effective coupling of the $f_{0}$ to kaons. Values in the literature vary from 2.51 [3] down to values compatible with zero [4]. An accurate measurement of $\Lambda$ therefore will strongly constrain $g_{f_{0} K \bar{K}}$. It should be stressed that in the couplings of physical particles to mesons important information about the nature of that particle is contained, as was shown by Weinberg for the case of the deuteron [5].

To our knowledge the impact of the CSB $f_{0}-a_{0}$ mixing on hadronic reactions close to the kaon threshold was studied in one microscopic model only [6]. There, the CSB

\footnotetext{
${ }^{2}$ Here we deviate from the original notation of Achasov et al. in order to introduce dimensionless coupling constants in line with the standard Flatté parameterization.

${ }^{3}$ Here we denote as common CSB effects those that occur at the Lagrangian level.
} 


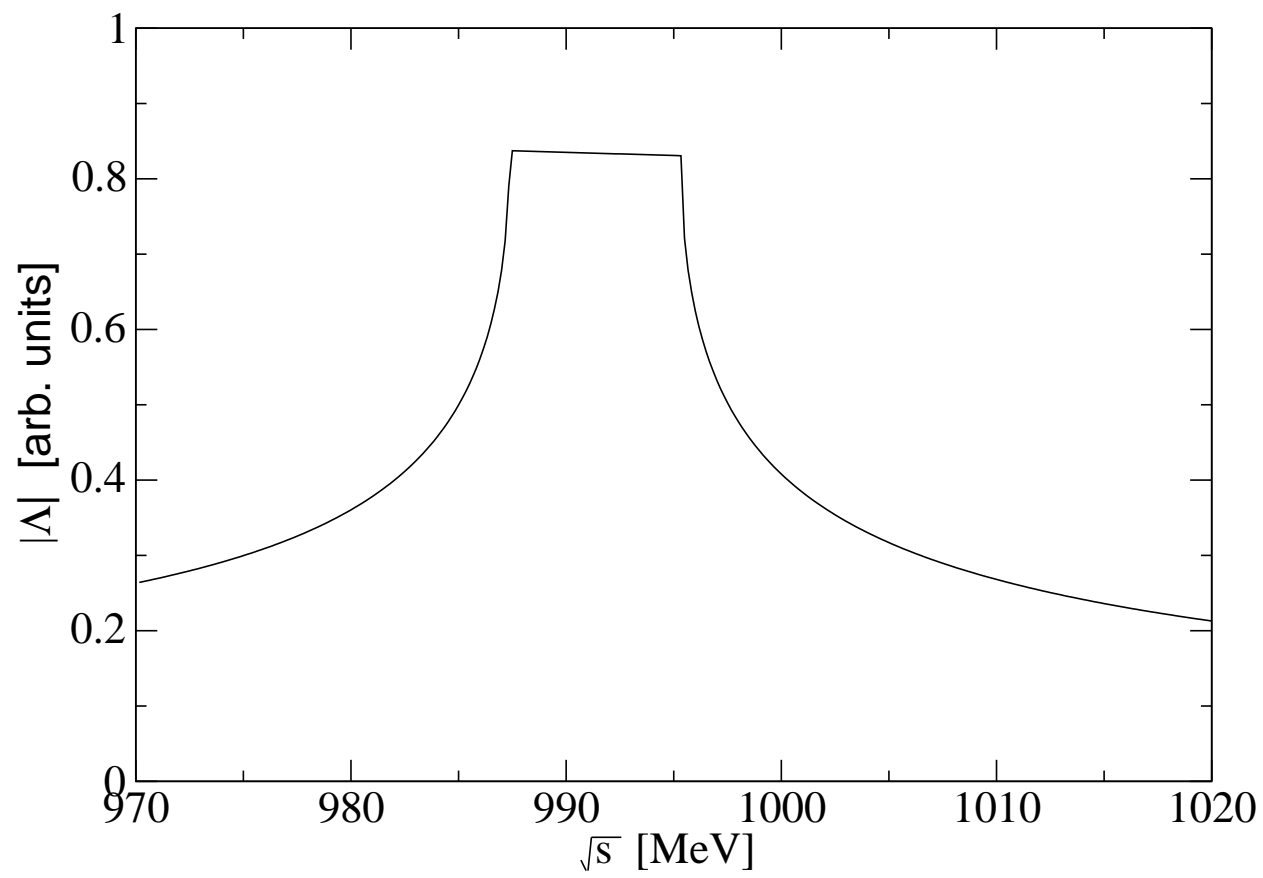

FIGURE 2. Modulus of the leading piece of the mixing amplitude $\Lambda$ defined in Eq. (1). The two kinks occur at the $K^{+} K^{-}$(at $987.35 \mathrm{MeV}$ ) and the $\bar{K}^{0} K^{0}(995.34 \mathrm{MeV})$ threshold respectively.

cross section $\pi^{+} \pi^{-} \rightarrow \pi^{0} \eta$ was predicted to be of the order of a few hundred $\mu b$ in line with the order of magnitude estimate derived from Eq. (11) ${ }^{4}$.

It is important to stress that $f_{0}-a_{0}$ mixing might have a significant impact also on the decay $\eta \rightarrow 3 \pi$ [7], however, in a very different kinematic regime compared to what we are looking at here. Thus one should expect that in addition to kaon loops also other CSB effects play a role. For a complete understanding of the $f_{0}-a_{0}$ mixing mechanisms knowledge about both kinematic regimes, that close to the resonance poles as well as that of the eta decay, is necessary.

With recent papers by Close and Kirk [8, 9] the interest in $a_{0}-f_{0}$ mixing was revived. Based on an analysis of central $p p$ collisions as well as radiative phi decays the authors extracted a mixing matrix element that was a factor of five larger than that given in Eq. (1) and independent of the invariant mass of the system produced. If confirmed, such a large mixing would indicate a completely different mechanism at work compared to what we believe in at present. Please note, that the work of Close and Kirk was heavily criticized in the literature [10, 11]. In any case, further experimental information is urgently called for.

In Ref. [12] it was stressed that a large mixing as that given by Eq. (1) should have a measurable impact on observables in photoproduction of scalar mesons. However, the signal will show up only as a modification of some observables and a good theoretical understanding of the rest of the amplitude is required to extract the strength of the mixing

${ }^{4}$ Here we assume the effective couplings to be of order 1. 
matrix element. In the next section we will argue that nucleon-nucleon induced reactions are especially suited to get insights on CSB and especially the $f_{0}-a_{0}$ mixing matrix element.

\section{THE SPECIAL FEATURES OF $N N$ AND $D D$ INDUCED REACTIONS}

For an unambiguous result for the mixing amplitude it is desirable to study observables that vanish in the absence of CSB. To identify such observables the production of scalars in $N N$ and $d d$ collisions is especially suited since these systems allows to manipulate the isospin. Especially when using deuterons as isospin filters, selection rules following from the symmetries of the strong interaction (parity, isospin) strongly restrict the behavior of various observables for these reactions. Therefore it is straight forward to study the violation of such symmetries in $N N$ and $d d$ collisions [13].

Experimentally so far the signals of CSB where extracted from $p n \rightarrow d \pi^{0}$ [14] and $d d \rightarrow \alpha \pi^{0}$ [15]. Since both the $\alpha$ particle as well as the deuteron are isoscalars whereas the pion is an isovector it is obvious that the latter reaction can only happen in the presence of CSB. To identify the signal from CSB in the former case one has to acknowledge that a $p n$ state, when being in a definite isospin state as it is in $p n \rightarrow$ $d \pi^{0}$ (the final state projects on isospin 1) behaves as a pair of identical particles as long as isospin is conserved. One obvious consequence is that the differential pion production cross section needs to be forward-backward symmetric. Thus any deviation from forward-backward symmetry is a signal of CSB. Analogously, one can look at a forward-backward asymmetry in $p n \rightarrow d a_{0}^{0}$ and the total cross section in $d d \rightarrow \alpha a_{0}^{0}$ by measuring a $\pi \eta$ pair in the final state at invariant masses of about $1 \mathrm{GeV}$ [16, 17] to study the scalar meson mixing. Note that $\pi \eta$ is the dominant decay channel of the $a_{0}$.

To extract and identify the relevant CSB mechanisms in the pion production reactions a detailed analysis within effective field theory is needed. The potentially most relevant sources of CSB currently discussed are isospin violating pion-nucleon scattering through the so called Weinberg term and $\pi-\eta$ mixing [15]. However, for a quantitative understanding of the cross sections measured still a lot of work from the theory side is needed.

In case of the production of scalar mesons the interpretation of a measured signal of CSB is more straight forward, since the resonances $a_{0}$ and $f_{0}$ are overlapping (the values for masses and widths given in the current version of the particle data booklet are basically equal [18]). Thus it should be intuitively clear that CSB in the propagation of the scalar mesons is kinematically favored as compared to CSB in the production operator. To make this statement more quantitative we compare the impact of $f_{0}-a_{0}$ mixing in the propagation of the scalar mesons (Fig. 3b) to that of $\pi-\eta$ mixing in the production operator (Fig. 3a). We regard the latter as a typical CSB effect and thus as a reasonable order of magnitude estimate for CSB in the production operator. Note, that the relevant dimensionless quantity for this comparison is the mixing matrix element times a propagator (c.f. Fig. 3). In the production operator the momentum transfer-at least close to the production threshold-is given by $t=-M_{N} m_{R}$, where $m_{R}$ denotes 


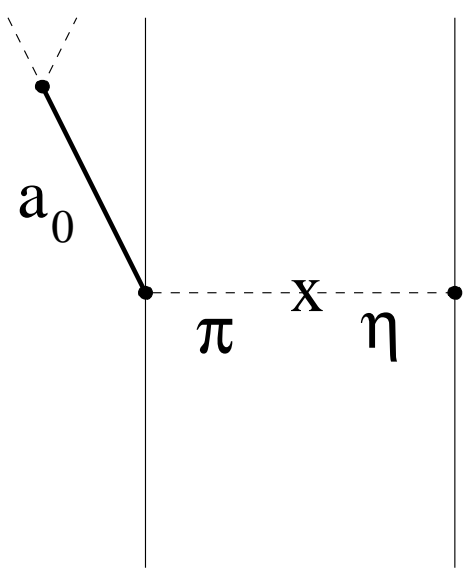

a)

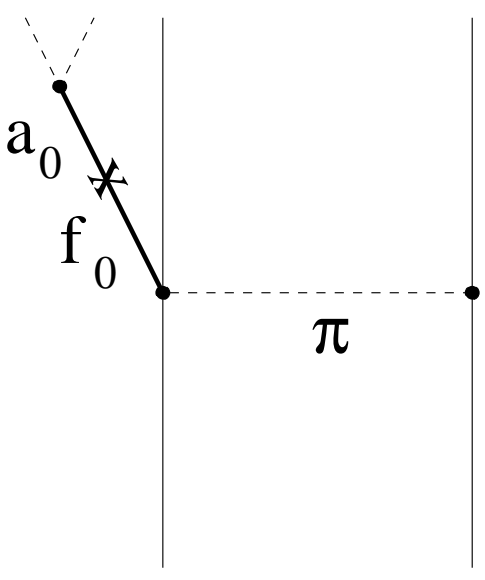

b)

FIGURE 3. Illustration of different sources of charge symmetry breaking: diagram a) shows CSB in the production operator through $\pi-\eta$ mixing and diagram b) shows CSB in the propagation of the scalars. Thin solid lines denote nucleons, thick solid lines scalar and dashed ones pseudoscalar mesons. The $X$ indicates the occurrence of a CSB matrix element.

the invariant mass of the meson system produced (or equivalently the mass of the resonance) and $M_{N}$ denotes the nucleon mass. Thus, the appearance of the $\eta$ propagator introduces a factor of about $1 / t$ into the amplitude, since $t \gg m_{\eta}^{2}$. On the other hand, the resonance propagator is given by $1 /\left(m_{R} \Gamma_{R}\right)$, as long as we concentrate on invariant masses of the outgoing meson system close to the resonance position. Here $\Gamma_{R}$ denotes the width of the scalar resonance. Thus we find using $\Gamma_{R}=50 \mathrm{MeV}$, that the CSB in the production operator is kinematically suppressed by a factor of more than $\Gamma_{R} / M_{N} \sim 1 / 20$ as compared to CSB in the propagation of the scalars. In addition, as was argued in the first section, the mixing matrix element of $f_{0}-a_{0}$ mixing as it occurs in the propagation of the scalars is enhanced. Given these two arguments we can be sure that CSB as it will be measured in $N N$ and $d d$ collisions close to the $K \bar{K}$ threshold will be dominated by $f_{0}-a_{0}$ mixing in the propagation of the scalars and we can assume the production operator as charge symmetry conserving.

There are two more reasons why looking at $f_{0}-a_{0}$ mixing in $p n \rightarrow d \pi^{0} \eta$ is particularly suited:

- the signal from mixing is kinematically enhanced [16] and

- the analyzing power gives a striking signal as well [19, 20].

To understand the first point we have to look in a little more detail into the selection rules for the reaction under discussion. If we assume isospin conservation the $p n$ system in the initial state has to fulfill the Pauli-Principle requiring $L+S+T$ being odd, where $L$, $S$ and $T$ denote the total angular momentum, spin and isospin of the initial state. Isospin conservation demands for an isovector (isoscalar) final state $L+S$ to be of even (odd) in the initial state. If we assume all final systems (the meson-meson system produced as well as the deuteron with respect to this) in an $s$-wave, then the final state has even parity and total angular momentum $J=1$, since the deuteron has $J_{d}=1$. Then parity 

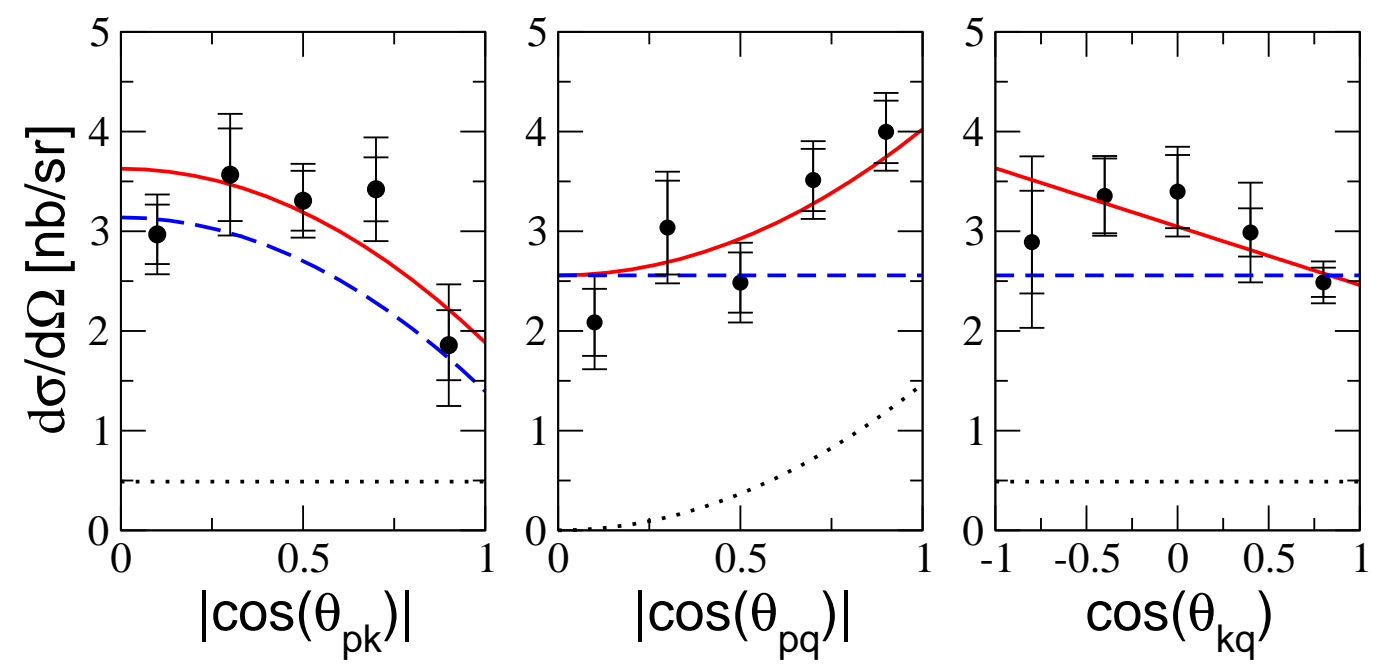

FIGURE 4. Angular distributions for the reaction $p p \rightarrow d \bar{K}^{0} K^{+}$. The solid line shows the result of the overall fit including both $\bar{K} K s$-wave as well as $p$-wave. To obtain the dashed (dotted) line the parameters for the $p$-wave ( $s$-wave) where set to zero (see text). The small error bars show the statistical uncertainty only, whereas the large ones contain both the systematic as well as the statistical uncertainty (c.f. Ref. [22]).

conservation demands the initial state to be even parity as well and thus we find that the overall $s$-wave in the final state calls for even parity in the initial state. Thus the production of an isovector needs an even parity $S=0$ state with $J=0$ as initial statethis does not exist. However, in the presence of CSB the $\pi \eta$ system might stem from an $f_{0}$ that in the propagation converts into an $a_{0}$. In this case the selection rules for the production of an isoscalar apply, calling for a $S=1$ even parity state with $J=1$. Thus both ${ }^{3} S_{1}$ and ${ }^{3} D_{1}$ are possible initial states leading to $s$-wave production of a scalar together with the deuteron. Therefore for kinematics close to the resonance poles the charge symmetry allowed amplitude is suppressed by a centrifugal barrier, whereas the charge-symmetry forbidden one is not.

To see that polarization observables and especially the analyzing power might be used as well one has to study in detail the corresponding amplitude structure. For this discussion we refer to Ref. [19]. However, it should be stressed that in contrast to the forward-backward asymmetry of the $\pi \eta$ system with respect to the deuteron, that can only occur in the presence of CSB, the corresponding signal in the analyzing power can also come from $p$-waves in the meson-meson system [20]. Fortunately one can study the contamination from meson-meson $p$-waves experimentally by studying the analyzing power in the corresponding charged channels $\left(\vec{p} p \rightarrow d \bar{K}^{0} K^{+}\right.$and $\left.\vec{p} p \rightarrow d \pi^{+} \eta\right)$ where there is no CSB. In this context it is reassuring that the $K \bar{K} p$-waves in the reaction $p p \rightarrow d \bar{K}^{0} K^{+}$are found to be small (c.f. next section) and the $\eta \pi p$-waves are estimated to be small [20, 21]. 

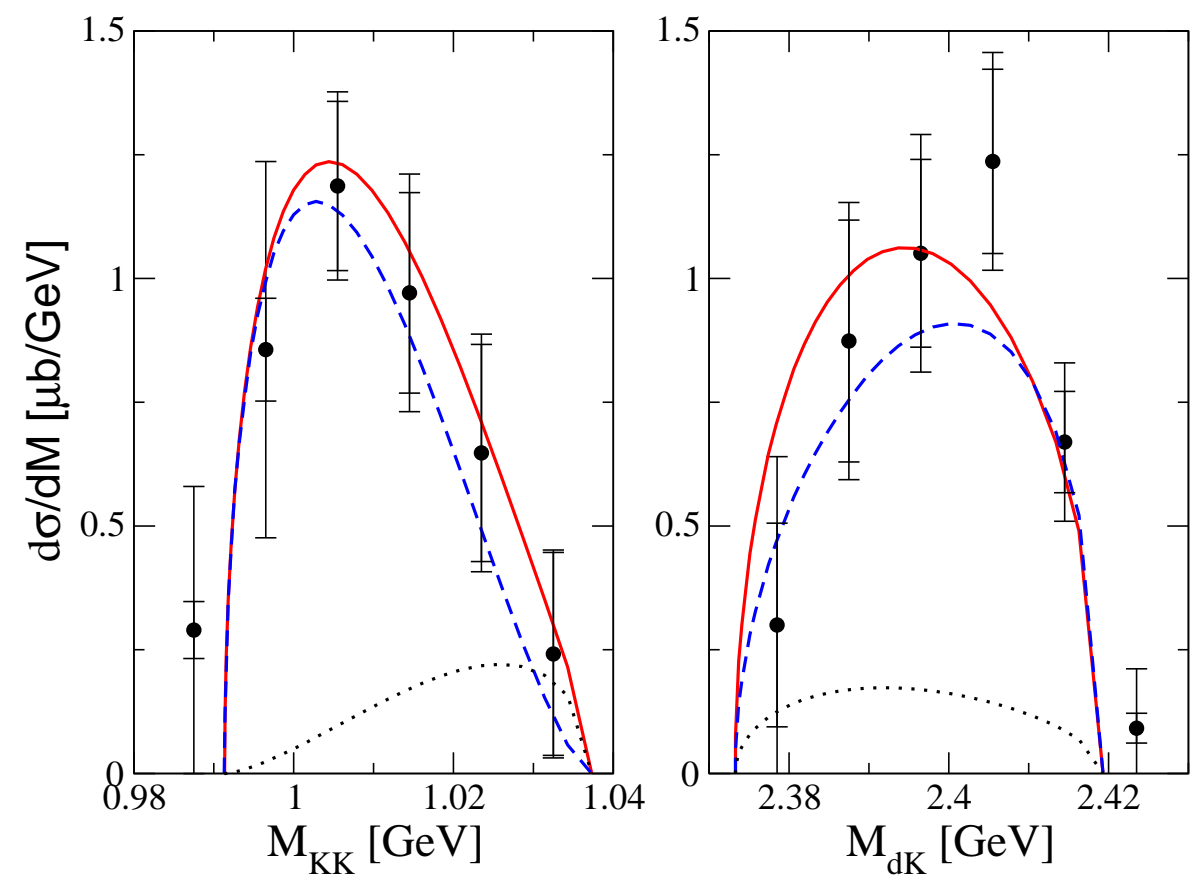

FIGURE 5. Various mass distributions for the reaction $p p \rightarrow d \bar{K}^{0} K^{+}$. Line code as in Fig. 44 The small error bars show the statistical uncertainty only, whereas the large ones contain both the systematic as well as the statistical uncertainty (c.f. Ref. [22]).

\section{THE REACTION $P P \rightarrow D \bar{K}^{0} K^{+}$}

Recently a first measurement of the reaction $p p \rightarrow d \bar{K}^{0} K^{+}$was reported close to the two kaon threshold [22] (the measurement was performed at an excess energy of only $46 \mathrm{MeV})$. In this section we will argue that, based on the experimental evidence together with very general assumptions, one has to conclude that the reaction $p p \rightarrow d \bar{K}^{0} K^{+}$is dominated by the scalar meson production in the final state. Thus, it is possible to study the scalar mesons in $N N$ induced reactions. This observation is not at all trivial, for there is another potentially strong final state interaction, namely that of the $\bar{K}^{0}$ with the deuteron that is enhanced due to the existence of the $\Lambda(1405)$, as was stressed in Ref. [21].

The data for three different angular distributions are shown in Fig. 4 those for two invariant mass distributions in Fig. 5. Note, the error bars are dominated by statistics! The data were fitted based on the assumption that either the $K \bar{K}$ system is in an $s$-wave while the deuteron with respect to this system is in an $p$-wave or the $K \bar{K}$ system is in a $p$-wave while the deuteron with respect to this system in in a $s$-wave (as shown in the previous section both subsystems in an $s$-wave simultaneously is not allowed). These assumptions lead to the following ansatz for the square of the spin averaged matrix 
TABLE 1. Result for the $C$ parameters from a fit to the experimental data. The parameters are given in units of $C_{0}^{k}$.

\begin{tabular}{ccccc}
\hline$C_{0}^{q}$ & $C_{0}^{k}$ & $C_{1}$ & $C_{2}$ & $C_{3}+\frac{1}{3} C_{4}$ \\
\hline $0 \pm 0.1$ & $1 \pm 0.03$ & $-0.6 \pm 0.1$ & $1.26 \pm 0.08$ & $-0.36 \pm 0.17$ \\
\hline
\end{tabular}

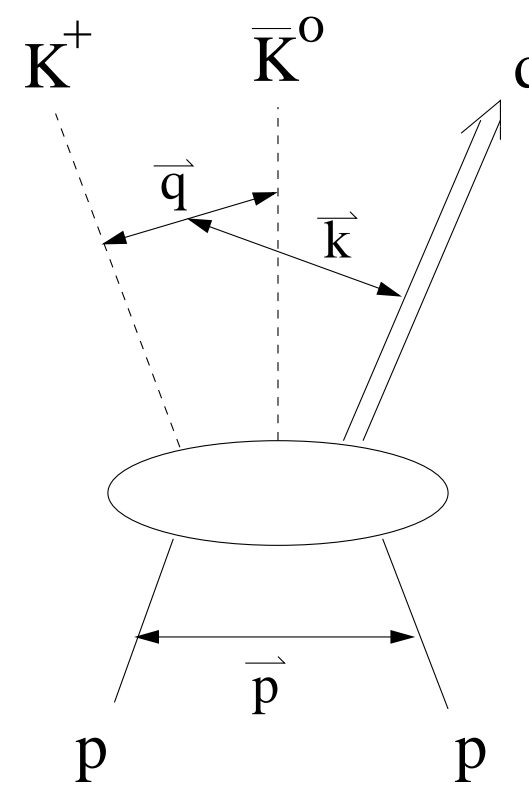

a)

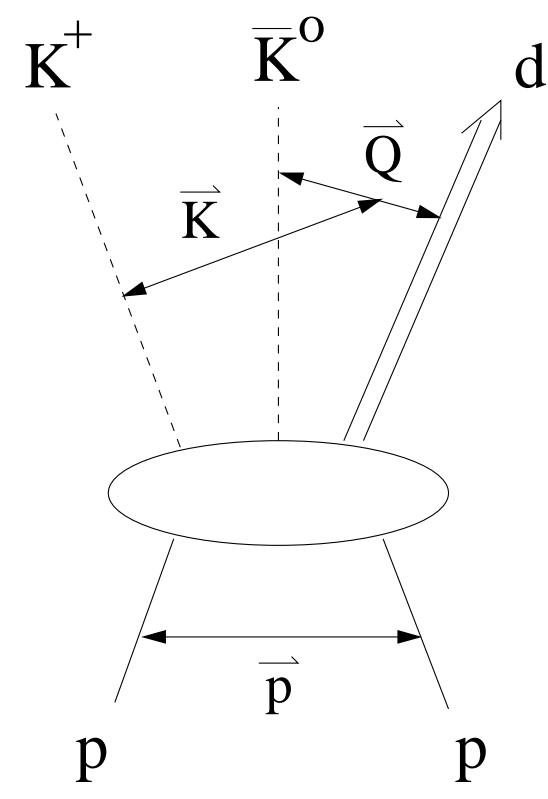

b)

FIGURE 6. Illustration of the coordinate system used in the analysis for the reaction $p p \rightarrow d \bar{K}^{0} K^{+}$.

element $[22]^{5}$ :

$$
|\bar{M}|^{2}=C_{0}^{q} q^{2}+C_{0}^{k} k^{2}+C_{1}(\vec{k} \cdot \hat{p})^{2}+C_{2}(\vec{q} \cdot \hat{p})^{2}+C_{3}(\vec{k} \cdot \vec{q})^{2}+C_{4}(\vec{k} \cdot \hat{p})(\vec{q} \cdot \hat{p})
$$

where $\hat{p}=\vec{p} /|\vec{p}|$ denotes the direction of the beam, $\vec{k}=\vec{p}_{d}$ is the relative momentum of the deuteron with respect to the $K \bar{K}$ system that at the same time agrees to the $\mathrm{cms}$ momentum of the deuteron, and $\vec{q}=\left(\vec{p}_{K^{+}}-\vec{p}_{K^{0}}\right) / 2$ denotes the relative momentum of the kaons. The coordinate system is illustrated in Fig. 6a). A fit to the data was carried out and the parameters found are given in Table 11. The result of the fit is shown in Figs. 4 and 5. The figures show not only the result of the overall fit of all parameters (solid line $)^{6}$, but also the fraction of the total result that stems from purely $K \bar{K} s$-waves (dashed-line) and from purely $K \bar{K} p$-waves (dotted-line). The data clearly show, that the dominant fraction of the data stem from $K \bar{K} s$-waves.

\footnotetext{
${ }^{5}$ Due to the proximity of the kaon threshold we use non-relativistic kinematics.

${ }^{6}$ Please note that the distributions given in Ref. [22] are not sensitive to $C_{3}$ and $C_{4}$ individually, but to the combination $C_{3}+(1 / 3) C_{4}$ only.
} 
To make the latter statement more quantitative one may use the contribution of the $K \bar{K} s$-waves to the total cross section as a measure of their significance. This leads to the following contributions:

$$
K \bar{K} s \text {-waves } 83 \% \quad \text { and } K \bar{K} p \text {-waves } 17 \%
$$

Since the pole that corresponds to the $a_{0}^{+}$is located close to the $\bar{K} K$ threshold, it will govern the propagation of the kaon pair in the $s$-wave. This is why in Ref. [22] it was claimed, that indeed the production of $a_{0}^{+}$was measured. In this context it is interesting to note that the $17 \%$ of $K \bar{K} p$-waves can be quantitatively understood as stemming from a $\pi \pi$ meson exchange current [23].

Since the excess energy in the $\bar{K} K d$ system produced is of the order of the width of the $a_{0}$, the latter does not show up as a bump in the invariant mass distributions [24] and its relevance can only be read of a stronger population of the corresponding partial wave. Note that, given the dominance of $K \bar{K} s$-waves, the $a_{0}$ should show up as a clear bump in the invariant mass spectrum for $p p \rightarrow d \eta \pi^{+}$, for here the corresponding threshold is far away.

In Ref. [21] it was argued that due to the resonant behavior of the $\bar{K} N$ systemthe hyperon resonance $\Lambda(1405)$ is situated close to the $\bar{K} N$ threshold-the $\bar{K} d$ final state interaction should play a significant role in the reaction dynamics. This final-state interaction should lead to an enhancement of the $\bar{K} d s$-wave. To see the amount of $\bar{K} d s-$ waves in the total cross section we have to change to coordinates that explicitly contain the $\bar{K} d$ relative momentum, as illustrated in Fig. 6b. Straight forward evaluation gives

$$
\vec{k}=\vec{Q}-\alpha \vec{K} \quad \text { and } \quad \vec{q}=\frac{1}{2}((2-\alpha) \vec{K}+\vec{Q}),
$$

where $\alpha=m_{d} /\left(m_{d}+m_{\bar{K}}\right)$. Given these expressions it is straight forward to re-express Eq. (2) in terms of $\vec{K}$ and $\vec{Q}$

$$
|\bar{M}|^{2}=B_{0}^{Q} Q^{2}+B_{0}^{K} K^{2}+B_{1}(\vec{K} \cdot \hat{p})^{2}+B_{2}(\vec{Q} \cdot \hat{p})^{2}+B_{3}(\vec{K} \cdot \vec{Q})^{2}+B_{4}(\vec{K} \cdot \hat{p})(\vec{Q} \cdot \hat{p}),
$$

where the various $B$-coefficients appearing are linear combinations of the $C$-coefficients of Eq. (2). E.g.

$$
B_{0}^{Q}=\frac{1}{4} C_{0}^{q}+C_{0}^{k}+\frac{1}{2} C_{3} \quad \text { and } \quad B_{2}=\frac{1}{4} C_{2}+C_{1}+\frac{1}{2} C_{4} .
$$

using the parameters of table 1 one finds the following contributions to the total production cross section:

$$
\bar{K} d s \text {-waves } 54 \% \quad \text { and } \quad \bar{K} d p \text {-waves } 46 \% \text {. }
$$

Thus the $\bar{K} d$ interaction does not play a prominent role in the reaction $p p \rightarrow d \bar{K}^{0} K^{+}$ close to the threshold. 


\section{SUMMARY AND OUTLOOK}

In this manuscript and the corresponding talk we stressed that

- $f_{0}-a_{0}$ mixing is a very interesting phenomenon to study,

- there is currently no unambiguous experimental signal for the existence of $f_{0}-a_{0}$ mixing,

- scalar meson production in $N N$ and $d d$ collisions are especially suited for this research,

- and first measurement of the reaction $p p \rightarrow d \bar{K}^{0} K^{+}$demonstrates the prominent role of scalar mesons in these production reactions.

For details on the current experimental program at the accelerator COSY we refer the interested reader to Ref. [2].

\section{ACKNOWLEDGMENTS}

Special thanks to A. Kudryartsev, V. Tarasov, J. Haidenbauer, and J. Speth for the collaboration that led to most of the results discussed here and to M. Büscher and V. Kleber for stimulating discussions and careful reading of the manuscript. I also would like to thank A. H. Fariborz for a workshop that scientifically as well as socially was extremely enjoyable.

\section{REFERENCES}

1. N.N. Achasov, S. D., and Shestakov, G., Phys. Lett., B88, 367-371 (1979).

2. Buscher, M., Sassen, F. P., Achasov, N. N., and Kondratyuk, L., hep-ph/0301126 (2003).

3. Achasov, M. N., et al., Phys. Lett., B485, 349-356 (2000).

4. Aitala, E. M., et al., Phys. Rev. Lett., 86, 765-769 (2001).

5. Weinberg, S., Phys. Rev., 130, 776 (1963).

6. Krehl, O., Rapp, R., and Speth, J., Phys. Lett., B390, 23-28 (1997).

7. Abdel-Rehim, A. M., Black, D., Fariborz, A. H., and Schechter, J., Phys. Rev., D67, 054001 (2003).

8. Close, F. E., and Kirk, A., Phys. Lett., B489, 24-28 (2000).

9. Close, F. E., and Kirk, A., Phys. Lett., B515, 13-16 (2001).

10. Achasov, N. N., and Kiselev, A. V., Phys. Lett., B534, 83-86 (2002).

11. Oller, J. A., Nucl. Phys., A714, 161-182 (2003).

12. Kerbikov, B., and Tabakin, F., Phys. Rev., C62, 064601 (2000).

13. Miller, G. A., Nefkens, B. M. K., and Slaus, I., Phys. Rept., 194, 1-116 (1990).

14. Opper, A., invited talk at the APS meeting (2003).

15. Stephenson, E. J., et al., nucl-ex/0305032 (2003).

16. Kudryavtsev, A. E., and Tarasov, V. E., JETP Lett., 72, 410-414 (2000).

17. Grishina, V. Y., Kondratyuk, L. A., Buscher, M., Cassing, W., and Stroher, H., Phys. Lett., B521, 217-224 (2001).

18. Hagiwara, K., et al., Phys. Rev., D66, 010001 (2002).

19. Kudryavtsev, A. E., Tarasov, V. E., Haidenbauer, J., Hanhart, C., and Speth, J., Phys. Rev., C66, 015207 (2002).

20. Kudryavtsev, A. E., Tarasov, V. E., Haidenbauer, J., Hanhart, C., and Speth, J., nucl-th/0304052 (2003). 
21. Oset, E., Oller, J. A., and Meissner, U.-G., Eur. Phys. J., A12, 435-446 (2001).

22. Kleber, V., et al., nucl-ex/0304020 (2003).

23. Grishina, V. Y., Kondratyuk, L. A., and Buscher, M., Krakow 2002, Production, properties and interaction of mesons, pp. 465-467 (2002).

24. Chernyshev, V. P., Fedorets, P. V., Kudryavtsev, A. E., and Tarasov, V. E., nucl-th/0110069 (2001). 